\title{
Shielding against SARS-Cov-2 infection is not justified in children with severe asthma
}

\author{
Michael Kabesch ${ }^{1}$ \\ ${ }^{1}$ University of Regensburg
}

July 20, 2020

To the Editor,

For the EU funded project PERMEABLE (PERsonalized MEdicine Approach for Asthma and Allergy Biologicals SeLEction), which addresses the availability of and access to advanced therapy of asthma in children across Europe, we performed a survey including 37 major pediatric asthma and allergy centers between September 2019 and July 2020. In total, the centers contributing to the survey treated approximately 1.000 young patients with severe asthma in 25 major European countries and Turkey with biologicals. In the light of the Corona Pandemic, we extended our survey asking the responsible clinicians if they experienced a SARS-CoV-2 infection in any of the children they are caring for. The questions pertaining to Corona infections were asked between March and July 2020.

Given the prevalence of SARS-CoV-2 infections in the general population and in children, one would expect that at least $1 \%$ of the patients would be affected (1). In fact, none of the centers was aware of any symptomatic COVID-19 case in their patient populations or any positive SARS-CoV-2 tests.

This leads to the conclusion, that either SARS-CoV-2 infections have a mild or even asymptomatic course also in children with severe asthma or that children with severe asthma (and their parents) were extremely successful in avoiding SARS-CoV-2 infections. Thus, we investigated by structured interview, how centers in those 26 countries had instructed their patients to avoid COVID-19. Interestingly, only 4 European countries (UK, Ireland, Portugal and Malta) had a strict, so called shielding policy in place which followed a principle of maximal segregation of severe asthmatics from the rest of the population: not leaving the house at all, not attending school even when they reopened, wearing face masks also at home, and social distancing even with family members. All other countries followed the principle of continuing or even enforcing asthma treatment in patients and advising to follow the same Corona rules as the general population.

Both strategies led to the same result: An absence of COVID-19 cases in children with severe asthma. We conclude from this observation, that shielding is not necessary in children with severe asthma as they and their families are perfectly able to avoid Corona infections. The harm done to children by enforcing seclusion, separation and stigmatization needs to be acknowledged. Deprivation of school, social contact and friends weights heavy on children and the absence of any COVID-19 cases in major European centers for severe asthma in children does not justify a policy of compulsory shielding of children with severe asthma, neither in the first nor in any further Corona wave.

Michael Kabesch, M.D.

1. University Children's Hospital Regensburg (KUNO) at the Hospital St. Hedwig of the Order of St. John, University of Regensburg, Regensburg, Germany.

2. Member of the Research and Development Campus Regensburg (WECARE) at the Hospital St. Hedwig of the Order of St. John, Regensburg, Germany. 


\section{References}

Stringhini S, Wisniak A, Piumatti G, et al. Seroprevalence of anti-SARS-CoV-2 IgG antibodies in Geneva, Switzerland (SEROCoV-POP): a population-based study [published online ahead of print, 2020 Jun 11]. Lancet . 2020;S0140-6736(20)31304-0. 